\title{
LOS PLANES DE ORDENAMIENTO TERRITORIAL, SUS CONTENDIENTES Y LOS NUEVOS INVITADOS
}

\section{PLANS OF REGIONAL PLANNING, ITS CONTENDERS AND NEW GUESTS}

\author{
CHAVOYA GAMA, Jorge Ignacio \\ Universidad de Guadalajara (UdeG) Centro Universitario de la Costa (CUC) \\ Departamento de Ciencias Exactas \\ Profesor Titular B \\ Av. Universidad 203 Delegación Ixtapa, C.P. 48280, Puerto Vallarta, Jalisco, México \\ Correo electrónico: jordinaqui@hotmail.com \\ Teléfono: +52 1-322-22-622-59
}

\section{RODRÍGUEZ ÁVALOS, Macedonio León}

Universidad de Guadalajara (UdeG) Centro Universitario de la Costa (CUC)

Departamento de Ciencias Exactas

Profesor Titular A

Av. Universidad 203 Delegación Ixtapa, C.P. 48280, Puerto Vallarta, Jalisco, México

Correo electrónico: leon.rguez@gmail.com

Teléfono: +52 1-322-22-622-59

Palabras Clave: Plan de Ordenamiento Territorial; Plan Estratégico; Proyecto Urbano

Key words: Plan of Regional Planning; Strategic Plan; Urban Project

\section{Resumen}

Partimos de iniciar el análisis y la discusión sobre los planes de ordenación del territorio y sus contendientes, iniciamos por hacer un breve análisis de los antecedentes de la planificación urbana en México para posteriormente revisar algunas de las transformaciones más significativas que las estructuras espaciales de las ciudades muestran, y como sé está respondiendo desde el campo de la planificación urbana. Como tercer paso se incluirá al tema del medio ambiente en términos de crisis hídrica y riesgo por cambio climático, y la desigualdad, como los nuevos "invitados" a tomar en cuenta, en la planeación; para finalizar con algunas reflexiones en torno a nuevos actores sobre la planeación normativa y la necesidad de trabajar sobre la planificación indicativa.

\begin{abstract}
We start the analysis and discussion about the management plans of the territory and its challengers, we start by making a brief analysis of the history of urban planning in Mexico for later review some of the most significant transformations that the spatial structures of the cities show is, and how it is answered from the field of urban planning. As a third step will include the issue of the environment in terms of water crisis, and climate change risk, and inequality, as the new "guests" to be taken into account, in planning; to conclude with some reflections on new actors on the normative planning and the need to work on the indicative planning.
\end{abstract}

Citación: ChavoYa GAMA, J. I. \& RODRíGueZ ÁVALOS, M. L. Los Planes de Ordenamiento Territorial, sus contendientes y los nuevos invitados. En: Libro de proceedings, CTV 2018. XII Congreso Internacional Ciudad y Territorio Virtual. "Ciudades y Territorios Inteligentes". UNCuyo, Mendoza, 5-7 septiembre 2018. Barcelona: CPSV, 2018, p. 15-28. 


\section{Introducción}

Las ciudades mexicanas, como la mayoría de Latinoamérica, padecen hoy en día un crecimiento acelerado de población, Incremento en el número de desempleados y subempleados reflejándose con mayor virulencia en el aumento del comercio informal y diversas formas de subsistencia, situación que paralelamente promueve la expansión física del área urbana hacia zonas naturales que las rodean y con ello el empeoramiento progresivo de las condiciones ambientales y de calidad de vida de la población, así como una creciente desigualdad.

Estas condiciones ponen de manifiesto una funcionalidad limitada de nuestras ciudades al presentar, entre otras características: saturación de las vialidades existentes, escasez de transporte público, con el consiguiente aumento en el tiempo destinado a los desplazamientos, déficit de los componentes del desarrollo urbano como suelo, infraestructura, vialidad y transporte, vivienda, equipamiento y servicios, medioambiente e insuficiencia en el abasto de alimentos y bienes de consumo, particularmente aquellos destinados a los sectores populares o de menores recursos.

Estas tendencias han provocado que se haya perdido la visión global de la ciudad, desvirtuándose el rol del plan como documento-intención de la colectividad urbana y ha conformado la elaboración de planes difícilmente ejecutables debido a la yuxtaposición de diferentes marcos normativos. Como consecuencia, han proliferado las disputas para la localización de equipamientos, infraestructuras o servicios, hecho que nos ejemplifica como el planeamiento no ha sido capaz de llevar a cabo su misión de establecer democráticamente un acuerdo general entorno a un proyecto de ciudad futura.

Las causas de los problemas, se ubican más allá de las manifestaciones físico- espaciales, ya que estas son los efectos de las desigualdades generadas por las recurrentes crisis económicas que han padecido la mayoría de los países latinoamericanos y a la heterogeneidad de las estructuras de la sociedad. Sin embargo, para muchos estudiosos de las ciudades latinoamericanas el proceso de construcción de nuestras ciudades puede y debe generar nuevas alternativas de desarrollo económico a través de los planes de ordenación del territorio, su gestión y acciones que de ellos se desprendan.

Para iniciar el análisis y la discusión sobre los planes de ordenación del territorio y sus contendientes, iniciaremos por hacer un breve análisis de los antecedentes de la planificación urbana para posteriormente comentar algunas de las transformaciones más significativas que las estructuras espaciales que las ciudades muestran y como sé está respondiendo desde el campo de la planificación urbana. Como tercer paso se incluirá al tema del medio ambiente en términos de crisis hídrica y riesgo por cambio climático, y la desigualdad, como los nuevos "invitados" a tomar en cuenta, en la planeación; para finalizar con algunas reflexiones en torno a nuevos actores sobre la planeación normativa y la necesidad de trabajar sobre la planificación indicativa.

\subsection{La planificación urbana en México}

Esta primera parte del artículo se divide de dos apartados. El primero comenta los antecedentes de la planificación urbana en el país y el segundo apartado versa sobre los esfuerzos actuales en esta materia:

Citación: CHAVOYA GAMA, J. I. \& RODRÍGUEZ ÁVALOS, M. L. Los Planes de Ordenamiento Territorial, sus contendientes y los nuevos invitados. En: Libro de proceedings, CTV 2018. XII Congreso Internacional Ciudad y Territorio Virtual. "Ciudades y Territorios Inteligentes". UNCuyo, Mendoza, 5-7 septiembre 2018. Barcelona: CPSV, 2018, p. 15-28. 


\subsubsection{Antecedentes de la planeación en México (1915 - 2018)}

Las políticas territoriales, urbanas y regionales empleadas en México a lo largo del siglo pasado $(\mathrm{XX})$ y en especial en las últimas décadas, experimentaron cambios importantes en su aplicación como parte de la evolución del país, algunas de ellas, como el Programa de 100 Ciudades lograron incidir de manera significativa en el periodo de 1992 al 2000.

Las políticas de desarrollo urbano pueden dividirse en cinco etapas en las que se pretendió establecer una adecuada administración integral del desarrollo urbano que se caracterizó por un desarrollo urbano y regional institucional sostenido más no estratégico:

a. La primera etapa, que comprendió de 1915 a 1940, destacó las políticas de desarrollo regional, la promulgación de las leyes de la Reforma Agraria en 1915 y la Constitución Política de Estados Unidos Mexicanos en 1917, dando como resultado con esta última, un conjunto de leyes federales que enriquecieron los instrumentos para la industrialización del país. (SPP. 1988)

b. De 1940 a 1976, comprende la segunda etapa, se impulsa el crecimiento económico y regional del país, mediante el surgimiento de políticas enfocadas al desarrollo regional y a la administración del territorio, favoreciendo con ello la industrialización de la capital del país. En 1946 se crean las Comisiones de Cuencas Hidrológicas, y con ello se dio inicio al desarrollo regional. (Garza. 1984)

c. En la tercera etapa, de 1976 a 1986, da inició la institucionalización de las políticas para el desarrollo regional, con la creación de la Secretaria de Asentamientos Humanos y Obras Públicas (SAHOP), se promueve la descentralización y desconcentración de la Zona Metropolitana del Valle de México, se promulga la Ley General de Asentamientos Humanos, donde se establecen las bases para la institucionalización del sector del desarrollo urbano. El Estado interviene en el proceso de la planeación del desarrollo, surgiendo con ello la planificación coordinada de los tres órdenes de gobierno en cuanto a las políticas crediticias, fiscales y las relativas a este nuevo sector de desarrollo urbano. (García. 2010) No obstante, la promulgación de la Ley General de Asentamientos Humanos y el establecimiento de los Convenios Únicos de Desarrollo (CUD), no se logró materializar las directrices para consolidar el desarrollo regional y la ordenación del territorio que permitiera la transformación de la economía nacional.

d. La cuarta etapa, de 1988 al 2003, se caracterizó por insertar a México en los mercados internacionales y la integración económica con los Estados Unidos de América, a través del TLC. En 1983 se crea la Secretaria de Desarrollo Urbano y Ecología (SEDUE), y se modifican los artículos constitucionales 27 y el 115. Con estas reformas se otorga a los municipios un mayor grado de responsabilidad en materia de desarrollo urbano, lo cual promovió la descentralización demográfica y administrativa hacia ciudades medias, de manera acelerada, apoyadas en la instrumentación del Programa de 100 Ciudades y consolidándose la institucionalización de las políticas de desarrollo urbano- regional. (García. 2010).

e. La quinta etapa, del 2006 al 2018 se ha caracterizado por tratar de redireccionar a México, hacia el cumplimiento de los acuerdos internacionales, para tal efecto se crea en 2012 la Secretaría de Desarrollo Agrario, Territorial y Urbano (SEDATU) encargada de llevar a cabo las políticas en torno a los objetivos del desarrollo sustentable, la nueva agenda 
urbana Hábitat III, el derecho a la ciudad como una política de justicia social, a la participación ciudadana, y a la vitalidad de las ciudades, en este periodo se han conformado ya 74 zonas metropolitanas, y el esfuerzo por ordenar el territorio y el desarrollo urbano, rápidamente ha evolucionado de manera vertiginosa, nuevos escenarios se presentan y nuevas leyes se ponen en vigencia, en 2012 se aprobó la ley de adaptación al cambio climático, donde se plantean directrices que deberán asumir los tres niveles de gobierno para afrontar estos nuevos retos ante el inminente embate del cambio climático, en 2016 se aprueba la nueva ley de asentamientos humanos, ordenamiento territorial y desarrollo urbano, como parte de la actualización y modernización de los esfuerzos por ordenar y regular el crecimiento urbano y reconocer como derecho fundamental el derecho a la ciudad lo que ha motivado a que los tres niveles de gobierno alinien y armonicen su legislación para hacer efectivo lo postulado en dichas leyes.

Estos esfuerzos de la política urbana, si bien han logrado algunos resultados positivos importantes como el impulso a las ciudades medias del país y el inicio de la descentralización del Gobierno Federal, lograron adicionalmente la transformación de la población del país: que pasó de una población eminentemente rural, a una población urbana, que aún no encuentra oportunidades o beneficios para permanecer en el campo.

Sin embargo, el resultado que se observa en la población residente en las ciudades tampoco es halagador, ya que es el de una población mayoritaria que vive en condiciones de pobreza y desigualdad social ubicada en zonas urbano-marginadas; una economía dominante de globalización, cuyos efectos se ven reflejados en segmentos de las ciudades y zonas metropolitanas y en los grupos de población que viven en esos segmentos.

En general existe una preocupación por la carencia de recursos y oportunidades, la falta de servicios y equipamientos adecuados para cumplir con las necesidades de la población que vive, trabaja y se divierte en las ciudades, y la necesidad de cuadros técnicos encargados de la planeación y la administración urbana, que deberían asesorar las decisiones políticas que operaran de manera poco eficiente los planes y programas de desarrollo urbano. Aunado a esta problemática, se observa la escasa participación de la población en la toma de decisiones responsables, la ejecución de acciones tendientes a resolver y minimizar los efectos ocasionados por la carencia de servicios de algunos los componentes del desarrollo urbano, suelo, infraestructura, vialidad y transporte, vivienda, equipamiento, medio ambiente, emergencias urbanas y servicios, situación que al agudizarse conlleva a transformar a las ciudades en entidades inseguras, disfuncionales e inhabilitables con un proceso de deterioro irreversible, repercutiendo en las condiciones de vida de toda la población.

\section{2 ¿Qué se hace actualmente?}

\subsubsection{Al nivel federal}

Se actualizó el Programa Nacional de Desarrollo Urbano y Ordenamiento del Territorio 2012 2018, el plantea 2 metas nacionales a). México incluyente, donde se alinean 4 objetivos y b). México seguro, donde se alinea 1 objetivo. Con líneas de acción o estrategias, así como políticas en que se estructura el programa: política de Ordenación del Territorio y Acción Urbana Regional; la política de Desarrollo Urbano y Regional; política de Suelo y Reserva y política de Prevención y Atención de Desastres. Con ellas se pretende maximizar la eficiencia económica del territorio, mediante la integración de un Sistema Urbano Nacional, así como la integración de suelo urbano apto el desarrollo.

Citación: CHAVOYA GAMA, J. I. \& RODRÍGUEZ ÁVALOS, M. L. Los Planes de Ordenamiento Territorial, sus contendientes y los nuevos invitados. En: Libro de proceedings, CTV 2018. XII Congreso Internacional Ciudad y Territorio Virtual. "Ciudades y Territorios Inteligentes". UNCuyo, Mendoza, 5-7 septiembre 2018. Barcelona: CPSV, 2018, p. 15-28. 
El programa se presentó en marzo del 2014 y propone nuevos enfoques y practicas sobre la planeación urbana y regional que permiten diseñar una política territorial que integre localidades rurales, pequeñas y dispersas en ámbitos regionales, y los barrios y zonas marginadas a las ciudades 0 a las grandes metrópolis a las que a pesar de no estar integradas en las mismas.

Es un esquema de planeación y actuación que busca combatir las causas estructurales de la pobreza y la marginación, que permite optimizar la eficiencia en el desarrollo urbano encaminado a elevar la competitividad económica de las ciudades y de regiones de país. Se pretende eslabonar el desarrollo en 5 ejes principales:

1) Fomentar ciudades compactas dosificando densidades de construcción, de tal forma que se mitigue la expansión urbana en zonas de alto valor ambiental y de riesgo.

2) Promover el derecho a la ciudad como un derecho colectivo a nivel constitucional y generar modificaciones a la legislación en la materia, para concebir la planeación territorial como un instrumento regulador único, incluyendo los niveles regionales y metropolitanos.

3) Generar instrumentos innovadores dirigidos a minimizar la fragmentación y segregación urbana, así como que coadyuven a generar vivienda dentro del tejido urbano para población de bajos recursos.

4) Establecer mecanismos para la recuperación de plusvalías de suelo a favor de la ciudad.

5) Promover crecimientos policéntricos y generar células urbanas con espacios que coadyuven a la convivencia social.

\subsubsection{Al nivel estatal y municipal}

Existen planes de diversas escalas y denominaciones: el plan estatal de desarrollo urbano; el plan o programa municipal de desarrollo; el plan o programa de desarrollo urbano de centro de población; los planes o programas parciales de desarrollo urbano; los planes o programas desarrollo urbano de puertos fronterizos, etc. y se encuentran en elaboración los planes o programas barriales de desarrollo urbano.

Todos ellos cuentan con términos de referencia proporcionados por la Secretaría de Desarrollo Agropecuario, Territorial y Urbano, que es la secretaria que cuenta con las atribuciones para ejercer y conducir la política de desarrollo urbano en el país. Todos los planes o programas de desarrollo urbano comparten en mayor o menor grado una estructura de seis partes, a saber: i). diagnóstico y pronóstico; ii). objetivos y metas; iii). normatividad; iv). Políticas y estrategias; v). programación y corresponsabilidad sectorial y vi). Instrumentación, seguimiento y retroalimentación.

\section{Los componentes de la estructura urbana}

La continua dinámica urbana, la complejidad de las funciones económicas, políticas y administrativas propias de la ciudad y la transformación tecnológica, han provocado diversas modificaciones en la organización del espacio físico.

Esta segunda parte del articulo considera algunos de los principales componentes de una estructura de ciudad: i). la ciudad (o metrópoli) en su conjunto ii). los distritos o colonias (barrios) y el corredor urbano y iii). la calle y el cuadrante (cuadra o manzana) y algunos ejemplos locales de cómo han sido estudiados y solucionados. 


\subsection{El nuevo reto, la ciudad-región metropolitana}

Es interesante observar que cuando nos referimos al término "ciudad" utilizamos un término que ni siquiera se encuentra definido legalmente. Nuestros planes de desarrollo urbano hacen referencia al centro de población o al municipio. Resulta paradójico que el término de ciudad hasta este momento lo hemos dejado de lado, cuando lo que observamos cómo proceso de transformación de ciudad en el territorio mexicano es el de un gran conjunto de centros de ciudad transformándose en ciudades metropolitanas o en regiones conformadas por un gran número de pequeños centros de población.

La nueva clasificación del Sistema Urbano Nacional al que hace referencia el Programa Nacional de Desarrollo Urbano y Ordenación del Territorio es de cinco clases de ciudades:

a. La megalópolis del centro, resultado de la "metropolización" de varias zonas metropolitanas y aglomeraciones urbanas en la región centro del país.

b. Zonas metropolitanas que son aquellas redes de ciudades donde los procesos de "metropolización" involucran a ciudades de México y de Estados Unidos de América o a ciudades de dos o más entidades federativas, así como aquellas grandes ciudades que tienen más de un millón de habitantes. Se subclasifican en: transfronterizas, interestatales y estatales.

c. Aglomeraciones urbanas que son las ciudades que han tenido procesos de expansión urbana hacia municipios adyacentes en la misma entidad federativa y tienen en conjunto una población inferior a un millón de habitantes.

d. Ciudades que son las que conforman localidades cuya expansión urbana no ha sobrepasado los límites del municipio donde se localizan. Esta clase se divide en 75 ciudades con más de 50,000 habitantes y en 244 ciudades entre 15,000 y 49,999 habitantes.

e. Las restantes 2,199 localidades del país entre 2,500 y 15,000 que se clasifican como centros de población.

Cabe hacer notar dos observaciones importantes:

En primer orden sólo las localidades menores caben en la clasificación de centros de población, el resto queda fuera de las jurisdicciones de sus propios municipios y por lo mismo queda fuera de los planes de desarrollo urbano. Las ciudades están desbordándose de sus límites políticos administrativos generando consigo políticas focalizadas y desintegradas, se están tomando decisiones y acciones para porciones de ciudad, y no para la totalidad, cuando se sabe que la competitividad de las ciudades es de orden regional o metropolitano si se pretende desarrollar economías acordes con la economía global.

La segunda observación es en cuanto a la dinámica de crecimiento de las ciudades de más de 100,000 habitante. En estudios recientes de CONAPO y SEDATU, se está llevando a cabo una reconsideración del término "zona metropolitana" y de las ciudades que deben entrar en esta nueva clasificación, ya que la llevada a cabo en el año 2006 para la presentación del Programa Nacional de Desarrollo Urbano ha quedado rebasada. Hoy se cuenta con más de 74 zonas metropolitanas en el país y será necesario considerar una reclasificación de sus planes de

Citación: Chavoya Gama, J. I. \& RODRíGueZ ÁVALOS, M. L. Los Planes de Ordenamiento Territorial, sus contendientes y los nuevos invitados. En: Libro de proceedings, CTV 2018. XII Congreso Internacional Ciudad y Territorio Virtual. "Ciudades y Territorios Inteligentes". UNCuyo, Mendoza, 5-7 septiembre 2018. Barcelona: CPSV, 2018, p. 15-28. 
desarrollo como consecuencia, ya que las ciudades metropolitanas, o áreas metropolitanas, o ciudades región, deben ser consideradas primordialmente como áreas sociopolíticas y económicas complejas y por ende su planeación debe darse bajo otra nueva óptica y otros objetivos.

\section{Los nuevos invitados: El medio ambiente (crisis hídrica), el riesgo- cambio climático y la desigualdad}

El tema del medio ambiente ha caminado con mayor velocidad y con mejores resultados en la conciencia ciudadana que el desarrollo urbano. Si bien el hombre se ha convertido en el mayor depredador del planeta, aún no hemos podido conciliar la política urbana como parte de la política del medio ambiente en general: el natural $y$ el construido. Este es el mayor reto que se nos antepone: ¿cómo conciliar las ciudades con el medio ambiente? Sin embargo, es un tema que no abordaremos porque sería motivo de otro artículo, pero no podemos dejar de lado un tema que seguimos sin abordar con la seriedad que le corresponde, pero aún lo hemos dejado fuera del ámbito de competencia de la planificación urbana: el tema del agua potable.

\subsection{El abastecimiento del agua potable}

De acuerdo con los estudiosos del tema, el abastecimiento del agua será el problema principal con el que se enfrentarán las ciudades en el futuro, es el problema que está para quedarse (Sánchez, 2017). Desgraciadamente el abastecimiento de agua a las ciudades se ha dejado en manos de medio ambientalistas $y$ de instituciones de orden nacional o regionales encargadas de la distribución del agua, totalmente desligado de las problemáticas de las comunidades y se ha optado por la solución más fácil: dejar que los organismos operadores de agua potable y de los organismos de suministro eléctrico tomen en última instancia las decisiones de permisos y licencias de construcción ya que la licencia se supedita a la factibilidad de obtener los servicios en mención y por consiguiente la verdadera decisión queda fuera de la de los urbanistas y tomadores de decisiones de las ciudades. Temas como la dirección o limitación al crecimiento o re-densificación de una ciudad están siendo consideradas en ese criterio.

La política de agua se vuelve uno de los temas que requieren de estudios $y$ análisis a profundidad, además de que requieren de acciones importantes a considerar. Algunos de estos temas identificados a lo largo de diversos análisis de ciudades son:

a. Las fuentes de abastecimiento internas y externas. Es muy común que se desconozcan fuentes internas como manantiales o pozos que son explotados en el ámbito privado por industrias o pozos que existen al interior de casas habitación que rara vez se registran.

b. Los sistemas de distribución, almacenamiento y rebombeo que presentan problemas comunes como:

- Deficiencia de las redes primarias (densidad mínima que no logra una presión satisfactoria en la red secundaria).

- Escasez en el suministro por la utilización de equipos obsoletos o falta de equipos.

- Generación de fugas por antigüedad de tuberías. Se han encontrado hasta 50 y $60 \%$ de desperdicio de agua en ciudades que presentan este problema.

- Generación de fugas por asentamiento del suelo, aun cuando las tuberías y los equipos son adecuados es importante considerar los cambios en desniveles particularmente en zonas sísmicas y de suelos poco compactos.

Citación: ChAVOYA GAMA, J. I. \& RODRíGUEZ ÁVALOS, M. L. Los Planes de Ordenamiento Territorial, sus contendientes y los nuevos invitados. En: Libro de proceedings, CTV 2018. XII Congreso Internacional Ciudad y Territorio Virtual. "Ciudades y Territorios Inteligentes". UNCuyo, Mendoza, 5-7 septiembre 2018. Barcelona: CPSV, 2018, p. 15-28. 
- Zonas de baja presión de agua especialmente en asentamientos de pendientes pronunciadas sin bombas de presión adecuadas.

- Zonas de suministro intermitente, $31 \%$ de habitantes.

- $30 \%$ de desperdicio del suministro en fugas.

c. La falta de sistemas circulatorios de reciclamiento de agua. Este tema aún no es considerado dentro de la normatividad de planes, y cuando se considera se ha planteado erróneamente y desarticulado de normas constructivas y arquitectónicas.

Paradójicamente un sistema de agua bien administrado y bien utilizado puede ser una fuente municipal de recursos importantes. Existen ejemplos interesantes como en las ciudades de León y Puerto Vallarta donde sus buenos resultados posibilitan su solución.

\subsubsection{Riesgo-Cambio climático e infraestructura critica}

El riesgo se construye socialmente y la gestión del riesgo es parte indisoluble de la gestión económica, social y política de las ciudades y su soporte territorial. Este tema presenta diversos componentes y enfoques que incluyen aspectos naturales, tecnológicos, sociales, ideológicos, culturales y organizativos, y requiere transitar por todo un proceso de trabajo apoyado por la voluntad política, en la búsqueda de soluciones o respuestas, bien sean de carácter estructural o no estructural, y la capacitación y la organización de la sociedad en pleno que garanticen la preparación para salvaguardar las pérdidas potenciales de vidas humanas, recursos económicos y el funcionamiento de la sociedad en su conjunto.

Los instrumentos de planeación urbana y territorial han estado en un constante cambio y perfeccionamiento al revalorizar sus cualidades e incorporar los novedosos aportes de la planificación estratégica, por ejemplo: la participación de todos los actores sociales en su ejecución y la utilización eficiente de los recursos humanos y tecnológicos disponibles y distribuidos espacialmente. En la actualidad, los planes de ordenamiento tienen como puntos focales la distribución espacial de las inversiones, el cambio de uso de suelo, el desarrollo del sistema de asentamientos humanos, la interpretación e integración de las políticas sectoriales, la búsqueda del equilibrio en el desarrollo, la elevación de la calidad de vida de la población, el uso racional de los recursos naturales y el mejoramiento de la calidad del medio ambiente, la eliminación de las desproporciones territoriales y la contribución a la disminución de la pobreza imperante en muchas regiones del planeta.

En estos esquemas de planeación todos los niveles toman decisiones a través de los planes de ordenamiento, donde en teoría deciden, en dónde, cual y cómo se distribuirá o concentrará la infraestructura para el buen funcionamiento de la ciudad. Estas decisiones de ordenamiento y política, dan forma a la infraestructura, influyen en el desarrollo socio-económico y no están exentas de ser expuestas a futuros peligros de índole diversa.

El cambio climático es un hecho concreto que tendrá repercusiones a escala global, el crecimiento de la población con el subsiguiente crecimiento de las ciudades, impulsan la demanda de nuevas infraestructuras y, como resultado, aumentan la exposición de la infraestructura a los peligros naturales. Se prevé que estas exposiciones a peligros naturales sean más grandes y más inciertas en el futuro debido a los efectos del aumento del nivel del mar y los cambios pronosticados en los patrones de temperatura y precipitación. (Brown 2014) Por lo tanto, la incorporación de la evaluación de riesgos de peligros naturales en la planificación de la infraestructura se está volviendo cada vez un desafío latente de importancia capital.

Citación: ChavoYa GamA, J. I. \& RODRíGUEZ ÁVALOS, M. L. Los Planes de Ordenamiento Territorial, sus contendientes y los nuevos invitados. En: Libro de proceedings, CTV 2018. XII Congreso Internacional Ciudad y Territorio Virtual. "Ciudades y Territorios Inteligentes". UNCuyo, Mendoza, 5-7 septiembre 2018. Barcelona: CPSV, 2018, p. 15-28. 
Retomando lo que plantea Hillary Brown en torno a la Infraestructura critica, que define como "como aquella cuyos activos, sistemas y redes, ya sean físicos o virtuales, son tan vitales para el país que su incapacidad o destrucción tendrían un efecto debilitante sobre la seguridad física, económica o pública, la salud, o cualquier combinación de estas". (Brown, 2014). Parte de identificar las regiones del país donde la infraestructura puede estar expuesta de manera única a un complejo conjunto de peligros naturales. En esas regiones, se destaca los tipos de infraestructura que están expuestas y los peligros que los ponen en riesgo. Cabe señalar que las exposiciones a peligros de la infraestructura siguen siendo muy inciertas, pero es de vital importancia incorporar esta categoría dentro de los planes de ordenamiento, tanto para mejorar la infraestructura y la resiliencia.

En la actualidad, la infraestructura en algunas áreas del país enfrenta una exposición desproporcionada a los peligros naturales, y es probable que esta exposición aumente en el futuro como resultado del cambio climático. Se necesita más información detallada sobre la exposición específica a los peligros naturales y la infraestructura especifica en una comunidad para responder de manera efectiva a los cambios en la exposición a los peligros naturales inducidos por el cambio climático.

Abordar los problemas de planificación efectiva e integral para mejorar la resiliencia contra la exposición actual y futura a peligros naturales de alta intensidad requerirá esfuerzos multifacéticos que incluyan recopilar información descriptiva de comunidades y sobre ellas, mejorar el conocimiento científico sobre fenómenos de riesgo y desarrollar herramientas e instituciones para planificar estrategias de mitigación para la serie compleja e incierta de riesgos naturales amenazan cada vez más a las comunidades en todo el país.

\subsubsection{La desigualdad}

El proceso global de neo-liberalización que, con características locales, se instauro en América Latina a inicios de la década de los ochentas, reconfiguro los espacios urbanos y los resignifico para la vida comunitaria. Mientras que por un lado los efectos económicos y sociales de las políticas de ajuste estructural golpeaban fuertemente a las poblaciones urbanas, por el otro las reformas del Estado trasladaron responsabilidades y autonomía a las administraciones municipales. Paralelamente se conectaron a los flujos globales produciendo un desdoblamiento de la articulación territorial de importantes dinámicas económicas, políticas y sociales hacia las ciudades (Carrión, 2016).

En este contexto donde los flujos de capital, bienes y servicios operan sobre el territorio de manera selectiva, las administraciones locales enfrentan una serie de obstáculos para constituirse como un verdadero contrapeso a las fuerzas y actores económicos e inmobiliarios. Además, la planificación ha pasado de centrarse en los planes como los instrumentos privilegiados para pensar la ciudad (con todas sus limitaciones) a una planificación parcializada que se centra en los "Grandes Proyectos Urbanos" como expresión de la selectividad del capital respecto al territorio (produciendo y reproduciendo mayor fragmentación socio-espacial (Carrión, 2016).

hoy la ciudad, se expresa como soporte de la mayor parte de las actividades humanas, es motivo de conflictos, entre las principales causas se encuentran: la escasez de suelo, objeto de demandas competitivas motivadas por fines económicos o sociales, la competencia de distintos usos sobre un mismo espacio, la sobreexplotación de los recursos naturales, la pugna entre el interés público y privado, la competencia entre sectores socioeconómicos por determinados

Citación: Chavoya Gama, J. I. \& RODRíGUEZ ÁvALOS, M. L. Los Planes de Ordenamiento Territorial, sus contendientes y los nuevos invitados. En: Libro de proceedings, CTV 2018. XII Congreso Internacional Ciudad y Territorio Virtual. "Ciudades y Territorios Inteligentes". UNCuyo, Mendoza, 5-7 septiembre 2018. Barcelona: CPSV, 2018, p. 15-28. 
recursos, las históricas contradicciones ciudad-ciudad y la contradicción entre conservación y desarrollo. Si bien en la actualidad se acepta el papel del ordenamiento urbano y territorial para lograr el correcto desarrollo socioeconómico, a veces en un espacio territorial específico se tropieza con intereses políticos o económicos muy arraigados y muchas veces difíciles de reconciliar con los objetivos de la organización territorial y la equidad social.

Cuando los intereses de los sectores hegemónicos de la sociedad están por encima del interés común, se contribuye a la concentración poblacional en las ciudades, al desarraigo de los habitantes de zonas rurales, a la conformación de cinturones precarios y segregados en las grandes ciudades, a la negligencia en los controles de actividades económicas de alto impacto ambiental y al aumento de desastres (Segura, 2014).

En el marco de estos procesos complejos, siguen existiendo y se han profundizado los obstáculos para la inclusión social, el acceso al "derecho a la ciudad" y la mejora en la calidad de vida. Los procesos de desigualdad urbana poseen distintas dimensiones que no se limitan a los estudios clásicos sobre segregación residencial, sino que es un tópico que guarda relación con las lógicas de producción del espacio urbano, el rol del capital y el tipo de modelo económico, y las pautas de interacción que se reproducen en la ciudad y configuran la experiencia urbana, los planes de ordenamiento enfrentan el reto de sobreponerse a los intereses especulativos y propiciar la reducción de la desigualdad.

\section{Lineamientos y proyectos estratégicos}

\subsection{La fundamentación de los planes}

Las bases jurídicas que dan sustento a la actualización de los planes y los elementos de enlace y congruencia con los diversos instrumentos de planeación en el ámbito nacional, estatal y municipal; son la Ley General de Asentamientos Humanos, Ordenamiento Territorial y Desarrollo Urbano, la Ley o Código de Desarrollo Urbano del Estado y los reglamentos estatales y municipales; a éstos hay que agregarles los elementos de enlace y congruencia con otras leyes como: las Leyes General y Estatal del Equilibrio Ecológico y la Protección al Ambiente: así como los lineamientos de otros niveles superiores de planeación: plan nacional de desarrollo, los programas nacionales de medio ambiente y los planes de desarrollo de los estados.

Todas estas subordinaciones y congruencias hacen que se tome muy difícil ya no solo elaborar un plan o programa de cualquier nivel sino el ¡Cómo llevarlo a la práctica! Las últimas décadas de planeación urbana nos han mostrado que de nada nos sirve articular planes de desarrollo si no llevamos a cabo una gestión que nos genere acciones y proyectos puntuales que se vuelvan los hacedores de los planes, de otra manera nos quedamos en el papel.

\subsection{De la planeación normativa a la planeación indicativa}

Las acciones prioritarias que concretarán el proyecto de ciudad deseada y que cuantificarán y programarán las instancias operativas responsables de aplicarlas, con base en recursos disponibles para los ejercicios presupuestales anuales emanadas de cualquier plan o programa de desarrollo urbano son mencionadas a continuación junto con algunas propuestas que las refuerzan en el ámbito de acciones específicas a manera de ejemplo:

Citación: CHAVOYA GAMA, J. I. \& RODRÍGUEZ ÁVALOS, M. L. Los Planes de Ordenamiento Territorial, sus contendientes y los nuevos invitados. En: Libro de proceedings, CTV 2018. XII Congreso Internacional Ciudad y Territorio Virtual. "Ciudades y Territorios Inteligentes". UNCuyo, Mendoza, 5-7 septiembre 2018. Barcelona: CPSV, 2018, p. 15-28. 
Las Acciones Estratégicas son incorporadas al programa o plan en los ámbitos generales y particulares y son de suma importancia ya que reflejarán acciones que podrán llevarse a cabo en el corto mediano o largo plazo, pero sobre todo generan la posibilidad de iniciar el proceso de planeación aquí y ahora con los recursos que puedan gestionarse en el mismo año que se dan a conocer los planes. Cabe mencionar que las acciones prioritarias contenidas generalmente responden a los ámbitos que se suscriben al proyecto de ciudad que queremos forjar.

Las acciones estratégicas establecidas en los planes o programas deben ser especificadas en el mismo programa y de ser posible darán los lineamientos para el desarrollo del proyecto arquitectónico, así como recomendaciones de los sitios adecuados y posibles para su construcción y operación.

Se considera que el programa o el plan responda eficientemente a las acciones prioritarias buscando que se logre una coordinación directa con grupos representativos de la ciudadanía y grupos profesionales que inciden en la planificación urbana tales como: consejos ciudadanos, agencias de desarrollo, institutos municipales de planeación, coplademunes, y otros que representen decisiones y visiones de la ciudad.

Aunado a las acciones estratégicas, los programas de acción deberán ser acompañados por los instrumentos de operación y los actores que puedan participar en la gestión, inversión, operación o mantenimiento de dichas acciones y de ser posible con los Programas Operativos Anuales de estados y municipios (POA's).

Dentro de las acciones estratégicas más comunes a implementar se encuentran:

- Proyectos de apoyo a la ampliación y renovación de la planta productiva y estímulo a la creación de empleo como proyectos adicionales a los corredores urbanos de servicios que permiten la diversificación de actividades y usos mixtos definidos como Habitación Mixta. En dichos corredores se permite el impulso al potencial de desarrollo económico, coadyuvando indirectamente a la creación de fuentes de empleo.

- Proyectos de equipamiento cultural recreativo, sobre todo en las zonas con mayor densidad habitacional y viviendas de baja calidad. Entre menor sea el espacio de vivienda, mayor y de mejor calidad deberá ser el proyecto cultural o recreativo.

- Aprovechamiento de la inversión acumulada y elevación de la calidad de vida, particularmente en los centros de ciudad y los centros históricos. Los centros de ciudad son áreas recreativas de acceso a toda la población que refuerzan la identidad y la calidad de vida de todos los habitantes.

- Proyectos de acceso equitativo a los servicios y autosuficiencia en el equipamiento local. La consolidación y optimización del uso de la infraestructura existente es una condición fundamental para mejorar la calidad de vida de las ciudades.

- Estructuración del territorio y ordenación del uso del suelo a través del aprovechamiento de los predios urbanos baldíos. Cada día la tendencia será a reutilizar y rediseñar nuestras ciudades para maximizar los servicios de infraestructura existente y evitar el continuo deterioro de las zonas con valor ecológico o agrícola.

- Conservación y reciclaje de la infraestructura y el medio construido. Se destaca la importancia del reciclamiento, conservación de infraestructura particularmente en áreas de uso habitacional e industrial y en especial el agua potable.

- Disminución de los desplazamientos y mejoramiento de la comunicación. Para está acción

Citación: ChavoYa GamA, J. I. \& RODRíGUEZ ÁVALOS, M. L. Los Planes de Ordenamiento Territorial, sus contendientes y los nuevos invitados. En: Libro de proceedings, CTV 2018. XII Congreso Internacional Ciudad y Territorio Virtual. "Ciudades y Territorios Inteligentes". UNCuyo, Mendoza, 5-7 septiembre 2018. Barcelona: CPSV, 2018, p. 15-28. 
estratégica las zonas Habitacionales con Comercio y algunas zonas Habitacionales Mixtas, así como corredores de localización urbanos a lo largo de arterias, que representan una alternativa de fuentes de empleo que permita arraigar a la población arraigar a la población económicamente activa, evitando desplazamientos innecesarios.

- Las acciones estratégicas específicas en los sistemas de transporte multimodales, así como cualquier alternativa al sistema de los niveles de operación de las vías principales de comunicación.

- De forma complementaria se han establecido acciones estratégicas claras para la conservación y regeneración de áreas verdes y espacios abiertos. Paralelamente, el cálculo de las áreas libres en colonias consolidadas y en proceso de consolidación tiene también la finalidad de aumentar la posible captación de agua pluvial para infiltración de los mantos acuíferos.

Adicionalmente cada vez son más las ciudades en el mundo que gestionan Grandes Proyectos Urbanos Integrales (GPUI) como estructuradores de sus planes o programas de desarrollo. La gestión eficiente, concertada, coordinada y democrática de las líneas estratégicas y de los grandes proyectos de desarrollo pueda llevarse a término y a la visión de ciudad que se planteen sus ciudadanos.

\section{Nuevos actores: en el proceso de toma de decisiones}

La planeación indicativa no tendría lugar sin la participación de los diversos actores que inciden en la construcción y reconstrucción de nuestras ciudades. Dentro de los instrumentos de participación ciudadana que existen dentro de la ley (cabe aclarar que habría que revisar que las Leyes estatales de participación ciudadana son vigentes); así como ciertos órganos administrativos de reciente creación que permiten canalizar las demandas de los ciudadanos para su atención se encuentran:

- El plebiscito a través del cual se puede consultar a los electores para que expresen su aprobación o rechazo previo a actos o decisiones de los presidentes municipales.

- El referéndum es un mecanismo de participación directa mediante el cual la ciudadanía manifiesta su aprobación o rechazo previo a una decisión de lo Asamblea Legislativo sobre la creación, modificación, reforma, derogación o abrogación de leyes respecto de materias de su competencia legislativa de esta última.

- La iniciativa popular es un mecanismo mediante el cual los ciudadanos podrán presentar a la Asamblea Legislativa, proyectos de creación, modificación, reforma, derogación o abrogación de leyes respecto de materias de su competencia y que lecorresponda a ésta expedir.

- En la consulta vecinal los vecinos de las demarcaciones territoriales podrán emitir opiniones y formular propuestas de solución a problemas colectivos del lugar donde residen.

- El presupuesto participativo es un mecanismo donde la autoridad municipal consulta a los vecinos sobre las obras prioritarias que demanda la colonia o barrio y esta priorización se refleja en el presupuesto de egresos de la administración y es vinculante para su ejecución.

En el caso de los órganos administrativos se encuentran aquellos que se encargan de gestionar, dar seguimiento e informar sobre las respuestas que proporcionan las áreas operativas a las solicitudes de servicios públicos proporcionados por los municipios en respuesta a los requerimientos de los ciudadanos.

Citación: ChAVOYA GAMA, J. I. \& RODRíGUEZ ÁVALOS, M. L. Los Planes de Ordenamiento Territorial, sus contendientes y los nuevos invitados. En: Libro de proceedings, CTV 2018. XII Congreso Internacional Ciudad y Territorio Virtual. "Ciudades y Territorios Inteligentes". UNCuyo, Mendoza, 5-7 septiembre 2018. Barcelona: CPSV, 2018, p. 15-28. 
Desgraciadamente la participación ciudadana en el desarrollo urbano a sido acotada a necesidades apremiantes y concretas de la población y pensando más en el interés de la comunidad cercana o individual que en un verdadero interés colectivo. Adicionalmente son cada vez más las autoridades que ven con recelo los procesos de participación ciudadana ya que usualmente son el inicio de bloqueos y negativas por parte de la población a proyectos 0 procesos generalmente no consultados inicialmente. Los procesos de participación ciudadana en nuestro país aún se encuentran en sus etapas tempranas, lejos de buscar unas formas integrales en las que autoridades y ciudadanía conformen estructuras organizadas eficientes en la toma de decisiones conjunta.

Sin embargo, México es un país con una larga tradición de participación ciudadana en la formación y conformación de las ciudades, particularmente en las zonas de pobladores de escasos recursos acostumbrados a tener que buscar sus propias alternativas ante la falta de respuestas por parte de las autoridades. Estos grupos van aprendiendo a ejercer presión sobre sus demandas y a complementarse con grupos adicionales que enfrentan necesidades comunes.

Existe una nueva corriente de participación ciudadana: la de grupos empresariales y profesionales que empiezan a interesarse en el desarrollo urbano como un proceso que empieza a afectar la calidad de vida de la ciudad en su totalidad y la de sus ciudadanos sin importar niveles socio-económicos. Estos grupos se manifiestan en la creación de institutos municipales de investigación y planeación y ya se ha generado una buena cantidad de experiencia que está evidenciando un rápido crecimiento con la incorporación de nuevos institutos. El fin último es la difusión y educación de la población de todas las edades y niveles socioeconómicos a que participen en los diversos ámbitos de acción de la ciudad. Desde la escuela, el trabajo, el barrio o la colonia, o desde los ámbitos de organizaciones profesionales se participe cotidiana y ampliamente.

\section{Conclusiones}

Son muchos y muy variados los planes o programas de desarrollo que tenemos a nuestro alcance, pero muy pocos los que se adaptan a las necesidades crecientes de las ciudades, particularmente las ciudades metropolitanas. Sin embargo, no podemos esperamos a que se transformen o modifiquen las estructuras legales, podemos avanzar en el empleo de enfoques y herramientas que nos ayudan a entender y a solucionar mejor nuestras ciudades en muy diversos temas competencia de los planes de desarrollo.

Desde el análisis socio-económico de la población, pasando por la consideración del cambio climático y el riesgo, contamos con nuevos enfoques metodológicos que debemos ajustar a las necesidades particulares de cada ciudad y cada estructura de ciudad. Sin embargo, podemos comparar problemas y modos de enfrentar esos problemas para llegar a soluciones únicas de acuerdo a cada ciudad, y cerrar la brecha de la desigualdad.

Finalmente, no podemos seguir definiendo o actualizando planes de desarrollo urbano sin proyectos estratégicos correspondientes que nos lleven a poner en marcha lo planeado, y sin invitar a la población a participar en todas y cada una de las etapas de la formación de los planes y de la consolidación de los mismos. Solo con una visión conjunta que compartan ciudadanos y autoridades podremos mejorar la calidad de vida de nuestras ciudades. De lo contrario todos los esfuerzos por realizar serán en vano.

Citación: ChavoYa GamA, J. I. \& RODRíGUEZ ÁVALOS, M. L. Los Planes de Ordenamiento Territorial, sus contendientes y los nuevos invitados. En: Libro de proceedings, CTV 2018. XII Congreso Internacional Ciudad y Territorio Virtual. "Ciudades y Territorios Inteligentes". UNCuyo, Mendoza, 5-7 septiembre 2018. Barcelona: CPSV, 2018, p. 15-28. 


\section{Bibliografía}

BROWN, H. Next Generation Infrastructure; Principles for Post-Industrial Public Works. Island Press, Washington D. C. USA. 2014.

CARRIÓN, F y DAMMERT, M. Los estudios urbanos en América Latina: un espejo donde mirarse. En: METZGER, P. et al. "La cuestión urbana en la región andina: miradas sobre la investigación y la formación". pp. 241-280, 2016. Pontificia Universidad Católica del Ecuador, Ecuador.

GARZA, G. Las políticas urbano-regionales en México (1915-1985). En: Pensamiento Iberoamericano, 2: 209-223. 1986. Instituto de Cooperación Iberoamericana, Madrid.

GARCÍA, F. La planeación del desarrollo regional en México (1900- 2006). En: Investigaciones Geográficas, Abril. 2010, 71, 102-121. [En línea] Disponible en: http://www.scielo.org.mx/scielo.php?script=sci arttext\&pid=S0188-46112010000100009

GOBIERNO FEDERAL. Plan Nacional de desarrollo Urbano 2012-2018. México.

LGAHOTDU. Ley General de Asentamientos Humanos, Ordenamiento Territorial y Desarrollo Urbano. Gobierno Federal México. 2016.

ONU-PNUD. Objetivos de Desarrollo Sostenible adoptados por México. 2015.

ONU-HABITAT. World Cities Report 2016, Urbanization and Development: Emerging, Futures. 2016.

ONU-HABITAT. Estado de las ciudades de América Latina y el Caribe 2012. Rumbo a una nueva transición urbana. Brasil: 2012.

ONU-HABITAT. Ciudades y el cambio climático: orientaciones para políticas informe mundial sobre asentamientos humanos. Brasil. 2011.

SÁNCHEZ, R. y Bonilla, A. (Eds.) Urbanización, Cambios Globales en el Ambiente y Desarrollo Sustentable en América Latina. Editorial Instituto Interamericano para la Investigación sobre Cambio Global - IAI. São José dos Campos; Brasil. 2017.

SEDATU. Secretaria de Desarrollo Agrario, Territorial y Urbano. México. 2017.

SEGURA, R. El espacio urbano y la (re) producción de desigualdades sociales. En: desiguALdades.net - Working Paper Series, 65: 1-31, 2014. [En línea] Disponible en: http://www.desigualdades.net/Resources/Working Paper/65-WP-Segura-Online.pdf?1393594017

SEMARNAT. Programa Sectorial de Medio Ambiente y Recursos Naturales. México. 2014.

SPP. SECRETARIA DE PROGRAMACIÓN Y PRESUPUESTO. Antología de la planeación en México 1917-1985. FCE, 20 Tomos, México. 1988.

Citación: Chavoya GamA, J. I. \& RODRíGUEZ ÁVALOS, M. L. Los Planes de Ordenamiento Territorial, sus contendientes y los nuevos invitados. En: Libro de proceedings, CTV 2018. XII Congreso Internacional Ciudad y Territorio Virtual. "Ciudades y Territorios Inteligentes". UNCuyo, Mendoza, 5-7 septiembre 2018. Barcelona: CPSV, 2018, p. 15-28. 